\title{
rpoB Mutations and Effects on Rifampin Resistance in Mycobacterium tuberculosis
}

\author{
Ma-chao Li, ${ }^{1} *$ jie Lu, $\mathbb{D}^{2, *}$ \\ Yao Lu, ${ }^{1,3, *}$ Tong-yang Xiao, (D) ${ }^{4, *}$ \\ Hai-can Liu, (iD) ${ }^{1}$ Shi-qiang Lin, ${ }^{5}$ \\ $\mathrm{Da}$ Xu, 'Gui-lian Li, ${ }^{\prime}$ \\ Xiu-qin Zhao,' Zhi-guang Liu, \\ Li-li Zhao,' Kang-lin Wan'
}

'State Key Laboratory of Infectious Disease Prevention and Control, National Institute for Communicable Disease Control and Prevention, Chinese Center for Disease Control and Prevention, Beijing, People's Republic of China; ${ }^{2}$ Beijing Key Laboratory for Pediatric Diseases of Otolaryngology, Head and Neck Surgery, MOE Key Laboratory of Major Diseases in Children, Beijing Pediatric Research Institute, Beijing Children's Hospital, Capital Medical University, National Center for Children's Health, Beijing, People's Republic of China; ${ }^{3}$ School of Laboratory Medicine and Life Sciences, Wenzhou Medical University, Wenzhou, People's Republic of China; ${ }^{4}$ Guangdong Key Laboratory for Diagnosis \& Treatment of Emerging Infectious Diseases, Shenzhen Third People's Hospital, Southern University of Science and Technology, Shenzhen, People's Republic of China; ${ }^{5}$ Department of Bioinformatics, College of Life Sciences, Fujian Agriculture and Forestry University, Fuzhou, People's Republic of China

*These authors contributed equally to this work

Correspondence: Li-li Zhao; Kang-lin Wan National Institute for Communicable Disease Control and Prevention, Chinese Center for Disease Control and Prevention, P.O. Box 5, Changping, Beijing, 102206,

People's Republic of China

$\mathrm{Tel} / \mathrm{Fax}+86 \quad 1058900779$

Email zhaolili@icdc.cn; wankanglin@icdc.cn
Objective: To investigate the mutations within the whole rpoB gene of Mycobacterium tuberculosis and analyze their effects on rifampin (RIF) resistance based on crystal structure. Methods: We sequenced the entire rpoB gene in 175 tuberculosis isolates and quantified their minimum inhibitory concentrations using microplate-based assays. Additionally, the structural interactions between wild-type/mutant RpoB and RIF were also analyzed.

Results: Results revealed that a total of 34 mutations distributed across 17 different sites within the whole rpoB gene were identified. Of the 34 mutations, 25 could alter the structural interaction between RpoB and RIF and contribute to RIF resistance. Statistical analysis showed that S450L, H445D, H445Y and H445R mutations were associated with high-level RIF resistance, while D435V was associated with moderate-level RIF resistance.

Conclusion: Some mutations within the $r p o B$ gene could affect the interaction between RpoB and RIF and thus are associated with RIF resistance. These findings could be helpful to design new antibiotics and develop novel diagnostic tools for drug resistance in TB.

Keywords: Mycobacterium tuberculosis, rifampin resistance, structure, mutation

\section{Introduction}

Although the incidence and mortality rates of tuberculosis (TB) are slowly declining, TB is still a major global public health threat with reports of $\sim 10$ million new cases and 1.2 million deaths annually. ${ }^{1}$ In recent years, there has been an alarming increase in drug-resistant TB, which poses a serious challenge for TB control. Rapid and accurate detection of drug resistance in TB patients is essential for the successful control of the disease.

Rifampin (RIF) is one of the most important anti-TB drugs. Its highly effective bactericidal activity against Mycobacterium tuberculosis (M. tuberculosis) has made it a principal drug in TB treatment. RIF targets the DNA-dependent RNA polymerase $\beta$-subunit, encoded by the rроB gene. ${ }^{2,3}$ Mutated $r p o B$ brings about a conformational change relevant to the binding affinity of RIF at $\beta$-subunit of the RNA polymerase (RNAP) and consequently the drug became inactive without proper binding to the target site. ${ }^{2,3}$ Approximately $90-95 \%$ of RIF-resistant isolates have been shown to harbor mutations within the $r p o B$ gene, ${ }^{4-6}$ and RIF resistance is largely associated with mutations in an 81-bp fragment of the rpoB gene, ${ }^{6-9}$ which located between rpoB codons 426 and 452 in M. tuberculosis. ${ }^{7,9-11}$ However, the mechanism of resistance concerning the rest 5\% RIF-resistant isolates is still unknown, indicating there might be other mechanisms, such as lowered cell wall permeability $^{12}$ or enhanced efflux pump. ${ }^{13,14}$ 
Prior reports suggest that particular mutations within $r p o B$ RRDR are more likely to confer higher levels of RIF resistance. ${ }^{5,15,16}$ Intriguingly, several rpo $B$ mutations were also observed in RIF-susceptible phenotypes. ${ }^{5,17,18}$ These contradictory results suggest that the association between RIF resistance and rpoB mutations should be explored in more detail for understanding the RIF resistance mechanism. Furthermore, the information on RIF resistance levels in $M$. tuberculosis strains without rpoB mutations is limited. In the present study, we systematically performed quantitative RIF resistance phenotyping with MIC measurements for $175 \mathrm{M}$. tuberculosis clinical isolates, and the mutations in the entire $r p o B$ gene and their effects on RIF resistance were also analyzed.

\section{Materials and Methods}

\section{M. tuberculosis Isolates}

A total of 175 stored M. tuberculosis clinical isolates (collected from 2005 to 2012) were included in this study. These isolates were obtained from 175 epidemiologically unrelated adult patients with pulmonary tuberculosis in China, of which $48.6 \%$ (85/175) were newly diagnosed cases. All isolates were cultured on LowensteinJensen (L-J) medium and freshly subcultured before being used for MIC testing.

\section{MIC Testing}

MICs were determined by in vitro 96-well microplatebased assay. Briefly, a $100 \mu \mathrm{L}$ volume of Middlebrook $7 \mathrm{H} 9$ broth was dispensed in each well of the plate. The drug concentrations tested for RIF were $0.125,0.25,0.5,1$, $2,4,8,16,32,64$ and 128. The inoculum was prepared from fresh L-J medium in $7 \mathrm{H} 9$ broth, then adjusted to a McFarland no. 1 turbidity standard and diluted 1:50. A $100 \mu \mathrm{L}$ volume was used to inoculate each well of the plate. Furthermore, drug-free (inoculum-only) controls were used to determine the time to read results. Plates were sealed and incubated at $37^{\circ} \mathrm{C}$ for approximately 7 10 days. After incubation, results were read using a Sensititre VIZION ${ }^{\circledR}$ system (Trek Diagnostic Systems, East Grinstead, WS, UK) Growth was evident as turbidity or as a deposit of cells at the bottom of the well. The MIC was recorded as the lowest concentration of antibiotics that inhibited visible growth. Control wells were evaluated first, and results were considered invalid when growth was not observed. The critical concentration was taken as
$1 \mu \mathrm{g} / \mathrm{mL}$ for RIF. ${ }^{19,20} \mathrm{H} 37 \mathrm{Rv}$ was used as a control with each batch of microplate-based assays.

\section{DNA Extraction, Amplification and Sequencing}

Genomic DNAs were extracted using the cetyltrimethylammonium bromide (CTAB) method ${ }^{21}$ and stored at $-20^{\circ} \mathrm{C}$ for further use.

The entire $r p o B$ gene was amplified using primers: $r p o B$ F1 (5'-GCGGCTCAGCGGTTTAGTTG-3'), rpoB-R1 (5'ACAGCGGGTTGTTCTGGTCC-3'), rpoB-F2 (5'-GACG ACATCGACCACTTC-3'), rpoB-R2 (5'-GATCACCTTG CCGGATTC-3'), rpoB-F3 (5'-CACATCGAGGAGCATGA $-3^{\prime}$ ), and $r p o B-\mathrm{R} 3$ (5'-CTCGCCATAGGACCATTG-3'). The cycling conditions were $94^{\circ} \mathrm{C}$ for $10 \mathrm{~min}$, followed by 35 cycles of $94^{\circ} \mathrm{C}$ for $1 \mathrm{~min}, 57^{\circ} \mathrm{C}$ for $40 \mathrm{sec}$, and $72^{\circ} \mathrm{C}$ for 50 sec, and a final extension at $72^{\circ} \mathrm{C}$ for $10 \mathrm{~min}$. All amplicons were purified, dried, and loaded onto an ABI 3730XL DNA analyzer (Applied Biosystems, Foster City, CA, USA). The sequences generated were aligned to the $\mathrm{H} 37 \mathrm{Rv}$ reference genome (GenBank accession number NC_000962) using BioEdit v7.05.3 (Tom A. Hall, Department of Microbiology, North Carolina State University, North Carolina, USA). All nucleotide sequences of $r p o B$ presented in the study were deposited in the NCBI SRA BioProject under the accession number: PRJNA722811.

\section{Quality Control}

Mixed peaks within a chromatogram and novel mutations were treated as true if they were reproducible. Furthermore, repeat testing was also performed on any isolate with a discrepancy between phenotypic susceptibility results and genotypic data.

\section{Structure Modelling and Analysis}

The complex structure of each RpoB mutant with RIF was modelled by SWISS-MODEL (https://swissmodel.expasy. org/) using Protein Data Bank (PDB) (http://www.rcsb. org/pdb/) file 5UHB as template. ${ }^{22}$ Structure analysis of protein-ligand complex was carried out by Discovery Studio Visualizer v.4.5 software (BIOVIA, Dassault Systèmes, San Diego, CA, USA). The interactions between wild-type/mutant RpoB and RIF were analyzed using the "Structure Monitor" and the 'Receptor-Ligand Interactions' modules. 


\section{Data Analysis}

The association of the rpoB mutation with the MICs was assessed using a regression multivariate model. $P$ value less than 0.01 was considered to be statistically significant. ${ }^{23,24}$ All data were performed using SAS version 9.4 (SAS Institute, Cary, NC, USA).

\section{Results}

\section{Mutations Within $r p o B$}

Among the 175 tested isolates, 125 (71.4\%) were resistant to RIF, and $50(28.6 \%)$ were susceptible to RIF (Table 1). In order to facilitate interpretation, MIC ranges were classified into three categories according to RIF resistance: low group (MIC 1-8 $\mu \mathrm{g} / \mathrm{mL}$ ), moderate group (MIC 16-64 $\mu \mathrm{g} / \mathrm{mL}$ ) and high group (MIC $\geq 128 \mu \mathrm{g} / \mathrm{mL})$. Among 125 RIF-resistant isolates, 19 (15.2\%), 18 (14.4\%) and 88 (70.4\%) had low, moderate and high levels of RIF resistance, respectively.

DNA sequencing showed that 118 isolates, including one susceptible isolate, 13 low-level resistant isolates, 18 moderate-level resistant isolates and 86 high-level resistant isolates, carried at least one non-synonymous mutation within the sequenced $r p o B$ gene (Table 1). Among them, 96 isolates $(81.4 \%)$ had a single mutation, while 22 isolates $(18.6 \%)$ had two mutations. For all mutations, 34 genotype patterns distributed across 17 different sites were detected, including 25 polymorphisms in the RRDR and nine polymorphisms outside the RRDR.

The most prevalent mutation was observed at codons 450,445 and 435 , which had mutated frequencies of $47.5 \%$ (56/118 isolates), $28.0 \%$ (33/118 isolates) and $16.1 \% \quad(19 / 118$ isolates $)$, respectively (Table 1). Ser450 was replaced by Leu (54 isolates), Phe ( 1 isolate) and Gly ( 1 isolate); His445 was replaced by Asp (11 isolates), Tyr (9 isolates), Arg (4 isolates), Asn (4 isolates), Leu (2 isolates), Cys (1 isolate), Gln (1 isolate) and Pro (1 isolate); Asp435 was replaced by Val (10 isolates), Gly (5 isolates), Tyr (3 isolates) and Glu (1 isolate). Other mutations were also observed at codons 45, 170, 400, 429, 430, 431, 432, 441, 446, 452, 488, 491, 759 and 1056, which had a total mutated frequency of only $27.1 \%$ (32/118 isolates). Notably, of these 32 isolates, 21 (65.6\%, 21/32 isolates) were combined with additional mutations at 435,445 and 450 . Moreover, two novel mutations (G759S and Q1056K) were identified in rpoB (Table 1).

\section{Association of $r p o B$ Mutations with RIF Resistance}

As $18.6 \%$ of isolates with rpoB mutations harbored more than one mutation, the association between mutations and drug resistance was estimated by multivariate regression (Table 2). In the multivariate model, the $r p o B$ mutations S450L, H445D, H445Y, and H445R were significantly associated with RIF resistance $(P<0.01)$, and isolates with these four mutations had a median MIC belonging to the high MIC category $(128 \mu \mathrm{g} / \mathrm{mL})$. Mutation D435V was also significantly correlated with RIF resistance $(P<0.01)$ according to multivariate analysis. Notably, D435V mutant had a median MIC of $64 \mu \mathrm{g} / \mathrm{mL}$, which was within the range of moderate MIC category.

Besides S450L, H445D, H445Y, H445R and D435V, there were some other mutations that were not identified by our multivariate analysis possibly due to small sample sizes. These mutations might also confer RIF resistance since our data showed that some isolates did not have the above-mentioned five mutations but were resistant to RIF. As shown in Tables 1 and 2, isolates within the moderate and high MIC category, harbored single mutation V170F, L430P, S441L, H445C, H445L, and Q432K, Q432L, Q432P, and S450F, respectively, as well as double mutations $\mathrm{V} 170 \mathrm{~F} / \mathrm{H} 445 \mathrm{~N}, \quad \mathrm{~L} 430 \mathrm{P} / \mathrm{S} 431 \mathrm{G}, \quad \mathrm{L} 430 \mathrm{R} / \mathrm{D} 435 \mathrm{G}$, L430P/D435G, D435G/L452P, D435G/I491N, and L430P/H445N, D435E/S441L, H445N/L452P, H445P/ $\mathrm{K} 446 \mathrm{Q}$, respectively. It was notable that one RIFsusceptible isolate ( $\mathrm{MIC}=0.5 \mu \mathrm{g} / \mathrm{mL}$ ) harbored single D435Y mutation. Another isolate that harbored the single D435Y mutation had a MIC of $1 \mu \mathrm{g} / \mathrm{mL}$. Finally, six lowlevel RIF-resistant isolates and two high-level RIFresistant isolates harbored no mutation in the whole rpoB gene.

\section{Changes of Intermolecular Interactions Between RpoB Mutants and RIF}

The effects of mutations on the interactions between RpoB mutants and RIF were explored using the crystal structure of RIF-RNAP complex. Figure 1A shows the detailed intermolecular interactions between wild-type RpoB and RIF. Overall, 17 residues of RpoB had mutations possible for RIF resistance, and the receptor-ligand interaction changes of mutations are summarized in Table 3.

Seven residues with mutations in this study do not directly interact with RIF in wild-type RpoB-RIF complex. Six residues, namely P45, T400, K446, I488, G759 and 
Table I Distribution of rpoB Mutations and MICs of Rifampin

\begin{tabular}{|c|c|c|c|c|c|c|c|c|c|c|c|c|}
\hline \multirow[t]{3}{*}{ Mutations in rров } & \multicolumn{11}{|c|}{ No. of Isolates } & \multirow{3}{*}{$\begin{array}{c}\text { Mutations Possibly Contribute to } \\
\text { Drug Resistance }\end{array}$} \\
\hline & \multicolumn{11}{|c|}{ MICs $(\mu g / m L)$} & \\
\hline & $\leq 0.125$ & 0.25 & 0.5 & $\mathbf{I}$ & 2 & 4 & 8 & 16 & 32 & 64 & $\geq \mathbf{1 2 8}$ & \\
\hline P45S, S450L & 0 & 0 & 0 & 0 & 0 & 0 & 0 & 0 & 0 & 0 & 1 & S450L \\
\hline VI70F & 0 & 0 & 0 & 0 & 0 & 0 & 0 & I & 0 & 0 & 0 & VI70F \\
\hline VI70F, H445N & 0 & 0 & 0 & 0 & 0 & 0 & 0 & I & 0 & 0 & 0 & VI70F, H445N \\
\hline T400I, H445Y & 0 & 0 & 0 & 0 & 0 & 0 & 0 & 0 & 0 & 0 & 1 & $\mathrm{H} 445 \mathrm{Y}$ \\
\hline Q429H, H445Y & 0 & 0 & 0 & 0 & 0 & 0 & 0 & 0 & 0 & 0 & I & $\mathrm{H} 445 \mathrm{Y}$ \\
\hline L430P & 0 & 0 & 0 & 0 & 0 & I & 0 & 0 & 0 & 0 & 0 & L430P \\
\hline L430P, S43IG & 0 & 0 & 0 & 0 & 0 & 0 & 0 & I & 0 & 0 & 0 & L430P \\
\hline L430R, D435G & 0 & 0 & 0 & 0 & 0 & 0 & I & 0 & 0 & 0 & 0 & L430R \\
\hline L430P, D435G & 0 & 0 & 0 & 0 & 0 & 0 & 0 & 0 & 1 & 0 & 0 & L430P \\
\hline L430P, H445N & 0 & 0 & 0 & 0 & 0 & 0 & 0 & 0 & 0 & 0 & 1 & L430P, H445N \\
\hline Q432K & 0 & 0 & 0 & 0 & 0 & 0 & 0 & 0 & 0 & 0 & 2 & Q432K \\
\hline Q432L & 0 & 0 & 0 & 0 & 0 & 0 & 0 & 0 & 0 & 0 & 2 & Q432L \\
\hline Q432P & 0 & 0 & 0 & 0 & 0 & 0 & 0 & 0 & 0 & 0 & 1 & Q432P \\
\hline D435E, S44IL & 0 & 0 & 0 & 0 & 0 & 0 & 0 & 0 & 0 & 0 & 1 & D435E, S44IL \\
\hline D435G, L452P & 0 & 0 & 0 & 0 & 0 & 0 & 0 & 0 & 0 & I & I & L452P \\
\hline D435G, I49IN & 0 & 0 & 0 & 0 & 0 & 0 & I & 0 & 0 & 0 & 0 & $149 \mid N$ \\
\hline D435V & 0 & 0 & 0 & 0 & 0 & 2 & 0 & 2 & 0 & 3 & 2 & D435V \\
\hline D435V, S450L & 0 & 0 & 0 & 0 & 0 & 0 & 0 & 0 & 0 & I & 0 & D435V, S450L \\
\hline D435Y & 0 & 0 & I & 1 & 0 & 0 & 0 & 0 & 0 & 0 & 0 & D435Y \\
\hline D435Y, S450G & 0 & 0 & 0 & 0 & 0 & 0 & 0 & I & 0 & 0 & 0 & D435Y, S450G \\
\hline S44IL & 0 & 0 & 0 & 0 & 0 & 0 & 0 & I & 0 & 0 & 0 & S44IL \\
\hline $\mathrm{H} 445 \mathrm{D}$ & 0 & 0 & 0 & 0 & 0 & 0 & 0 & 0 & 0 & 0 & 11 & $\mathrm{H} 445 \mathrm{D}$ \\
\hline $\mathrm{H} 445 \mathrm{~L}$ & 0 & 0 & 0 & 0 & I & I & 0 & 0 & 0 & 0 & 0 & $\mathrm{H} 445 \mathrm{~L}$ \\
\hline $\mathrm{H} 445 \mathrm{~N}, \mathrm{~L} 452 \mathrm{P}$ & 0 & 0 & 0 & 0 & 0 & 0 & 0 & 0 & 0 & 0 & 2 & $\mathrm{H} 445 \mathrm{~N}, \mathrm{~L} 452 \mathrm{P}$ \\
\hline H445P, K446Q & 0 & 0 & 0 & 0 & 0 & 0 & 0 & 0 & 0 & 0 & 1 & $\mathrm{H} 445 \mathrm{P}$ \\
\hline H445Q, I49IM & 0 & 0 & 0 & 0 & 0 & 0 & I & 0 & 0 & 0 & 0 & H445Q, I49IM \\
\hline $\mathrm{H} 445 \mathrm{R}$ & 0 & 0 & 0 & 0 & 0 & 0 & 0 & 0 & 0 & 0 & 4 & $\mathrm{H} 445 \mathrm{R}$ \\
\hline $\mathrm{H} 445 \mathrm{C}$ & 0 & 0 & 0 & 0 & I & 0 & 0 & 0 & 0 & 0 & 0 & $\mathrm{H} 445 \mathrm{C}$ \\
\hline $\mathrm{H} 445 \mathrm{Y}$ & 0 & 0 & 0 & 0 & 0 & 0 & 0 & I & 0 & 0 & 6 & $\mathrm{H} 445 \mathrm{Y}$ \\
\hline S450F & 0 & 0 & 0 & 0 & 0 & 0 & 0 & 0 & 0 & 0 & 1 & $\mathrm{~S} 450 \mathrm{~F}$ \\
\hline S450L & 0 & 0 & 0 & 0 & 0 & 0 & 0 & 0 & I & 3 & 44 & S450L \\
\hline S450L, I488V & 0 & 0 & 0 & 0 & 0 & 0 & 0 & 0 & 0 & 0 & I & S450L \\
\hline S450L, I49IV & 0 & 0 & 0 & 0 & 0 & 0 & 0 & 0 & 0 & 0 & 1 & S450L, I49IV \\
\hline S450L, G759S* & 0 & 0 & 0 & 0 & 0 & 0 & 0 & 0 & 0 & 0 & 1 & S450L \\
\hline S450L, Q1056K* & 0 & 0 & 0 & 0 & 0 & 0 & 0 & 0 & 0 & 0 & 1 & S450L \\
\hline L452P & 0 & 0 & 0 & I & 2 & 0 & 0 & 0 & 0 & 0 & 0 & L452P \\
\hline No mutation & 40 & 7 & 2 & 3 & I & I & 1 & 0 & 0 & 0 & 2 & - \\
\hline
\end{tabular}

Note: *Mutation reported for the first time.

Q1056 are not involved in RIF binding, and the mutations of these residues do not have the interaction with RIF (Table 3). The other residue, S441, does not have any direct interaction with RIF in wild-type RpoB-RIF complex, but S441L mutation introduces steric hindrance between L441 and H445, leading to the loss of hydrogen bond between H445 and RIF (Figure 1B). Notably, the double mutant of D435E/S441L not only loses the hydrogen bond between H445 and RIF but also introduces steric hindrance between E435 and RIF (Figure 1C).

The other 10 residues directly interact with RIF, namely V170, Q429, L430, S431, Q432, D435, H445, S450, L452 and I491 (Figure 1A). Residues Q432, H445 and S450 have intermolecular hydrogen bonds with RIF. Residues L430, L452 and I491 participate in hydrophobic interactions between RpoB and RIF. Residues V170, 
Table 2 Logistic Regression Multivariate Model Results Between rpoB Mutations and RIF Resistance

\begin{tabular}{|c|c|c|c|}
\hline Mutation & No. of Isolates & Median MIC (IQR) & $P$ value \\
\hline Q432K & 2 & $128(128,128)$ & 0.104 \\
\hline Q432L & 2 & $128(128,128)$ & 0.104 \\
\hline $\mathrm{H} 445 \mathrm{~N}$ & 4 & $128(72,128)$ & I \\
\hline H445R & 4 & $128(128,128)$ & $0.007^{\mathrm{a}}$ \\
\hline H445L & 2 & $3(2,4)$ & 0.104 \\
\hline H445D & 11 & $128(128,128)$ & $<0.000^{\mathrm{a}}$ \\
\hline $\mathrm{H} 445 \mathrm{Y}$ & 9 & $128(128,128)$ & $<0.000^{\mathrm{a}}$ \\
\hline L430P & 4 & $24(10,80)$ & 0.104 \\
\hline L452P & 7 & $64(2,128)$ & 0.026 \\
\hline D435G & 5 & $32(8,64)$ & 0.104 \\
\hline D435V & 10 & $64(16,64)$ & $<0.000^{\mathrm{a}}$ \\
\hline D435Y & 3 & I $(0.5,16)$ & 0.261 \\
\hline VI70F & 2 & $16(16,16)$ & 0.444 \\
\hline S44IL & 2 & $72(16,128)$ & 0.104 \\
\hline S450L & 54 & $128(128,128)$ & $<0.000^{\mathrm{a}}$ \\
\hline
\end{tabular}

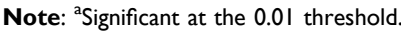

Abbreviation: IQR, interquartile range.

Q429, D435 and S431 involve in van der Waals interactions.

Structural studies reveal that intermolecular hydrogen bonds are essential for the binding of RIF, ${ }^{2,25}$ and H445 and S450 are the crucial residues for the intermolecular hydrogen bonds. As shown in Figure 2, the eight mutations of H445 all lead to loss of hydrogen bond, and H445Y mutation additionally introduces steric hindrance; thus, all the mutations of $\mathrm{H} 445$ contribute to RIF resistance in TB. For residue 450, S450G only loses the intermolecular hydrogen bond, while S450L and $\mathrm{S} 450 \mathrm{~F}$ result in both loss of hydrogen bond and introduction of steric hindrance (Figure 3). Moreover, Q432 also participates in the formation of intermolecular hydrogen bonds, and Q432P and Q432L lead to loss of hydrogen bond and confer RIF resistance in TB. Mutations of hydrophobic residues L430, L452 and I491 result in loss of hydrophobic interaction between RpoB and RIF, leading to RIF resistance as well. In addition, some mutations introduce steric hindrance and electrostatic repulsion in RpoB-RIF complex, which are unfavorable for RIF binding. However, mutations Q429H, S431G and D435G do not change the intermolecular interactions between wild-type RpoB and RIF (Table 3).

\section{Discussion}

Rapid and accurate detection of drug resistance in M. tuberculosis is essential for improving treatment success and decreasing the spread of disease. In recent years, several molecular diagnostics techniques, such as GeneXpert MTB/RIF and Geno Type MTBDRplus, have been developed to allow the rapid identification of M. tuberculosis drug resistance directly from specimens. $^{26-28}$ However, these molecular methods can miss isolates with mutations outside the target region, and cannot determine the level of resistance. In addition, several reports suggest that some isolates with $r p o B$ mutations are RIF-susceptible according to culture-based phenotypic drug susceptibility testing. ${ }^{4,5,18,29}$ Previous studies indicate that RIF resistance is a surrogate marker of MDR$\mathrm{TB},{ }^{4,5}$ hence rapid and accurate detection of RIF resistance is important.

It is known that the $\beta$-subunit of DNA-dependent RNA polymerase encoded by $r p o B$ is a target for rifamycin drugs, and that amino acid changes in this protein can confer rifamycin resistance. $9,10,30$ Our current results
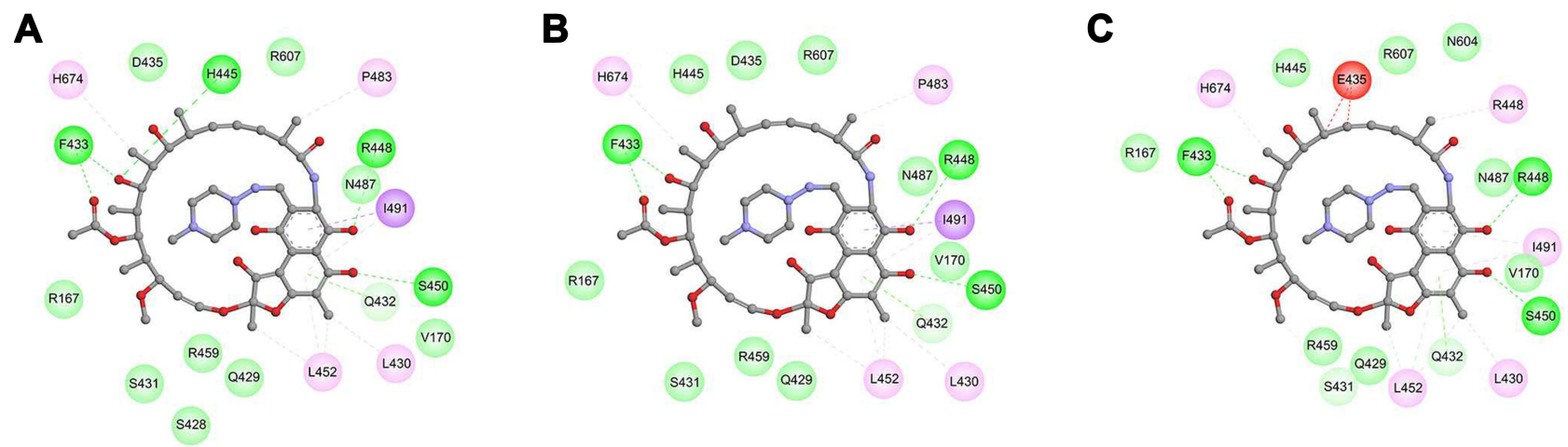

Figure I The 2D diagram showing the interactions between rifampin and wild-type RpoB (A), mutant S44IL (B) or double mutant D435E \& S44IL (C). The rifampin (RIF) molecule is shown in the middle with a display style of ball and stick. The colored balls around RIF molecule indicate the residues involved in the direct interactions between RpoB and RIF. The green, magenta and red dash lines connecting RIF and corresponding residues indicate intermolecular hydrogen bonds, hydrophobic interactions and steric hindrance, respectively. Residues involved in van der Waals interactions are represented by light green balls without any dash line linked to RIF. 
Table 3 Summary for Interaction Changes of Mutations Based on Structural Analysis

\begin{tabular}{|l|l|}
\hline Interaction Changes & Mutations \\
\hline No interaction with RIF & P45S, T400I, K446Q, I488V, G759S, QI056K \\
\hline No change & Q429H, S43IG, D435G \\
\hline Hydrogen bond lost & Q432P, Q432L, H445R, H445L, H445Y, H445C, H445D, H445P, H445N, H445Q, S450L, S450F, S450G \\
\hline Hydrophobic interaction lost & L430P, L430R, L452P, I49IN, I49IV \\
\hline Steric hindrance & VI70F, Q432L, D435V, D435E, D435Y, S44IL, H445Y, S450L, S450F, L452P, I49IM \\
\hline Electrostatic repulsion & Q432K \\
\hline
\end{tabular}

revealed that $93.6 \%(117 / 125$ isolates $)$ of RIF-resistant isolates carried at least one mutation in rpoB. Overall, 17 distinct codons in $r p o B$ had mutations contributing to amino acid replacements, of which 10 codons are located in the RRDR and seven are located outside the RRDR. Interestingly, almost all mutations outside the RRDR were accompanied by mutations within the RRDR. These results indicate the potential for using DNA sequencing of RRDR to rapidly predict RIF. ${ }^{4,7}$

As evidenced in a number of studies, the most frequently mutated codons associated with RIF in the $r p o B$ gene are codons 435, 445 and 450. ${ }^{4,5,16,31,32}$ The reported frequencies of these three mutations from world geographic regions range from $1.1 \%$ to $20.4 \%$ for codon 435 , from $6.8 \%$ to $32 \%$ for codon 445 and from $31 \%$ to $80.9 \%$ for codon 450 , respectively. ${ }^{4,6,7,33,34}$ In this study, the majority of mutations in RIF-resistant isolates were at codons 435, 445 and 450, with the mutation frequencies of $16.1 \%, 28.0 \%$ and $47.5 \%$, respectively. The levels of RIF resistance in isolates with rрo $B$ mutations at these three codons were observed to be dependent on the amino acid change. In multivariate analysis, S450L, H445D, H445Y and H445R were strongly associated with high-level RIF resistance; D435V was strongly associated with moderate-level RIF resistance. ${ }^{5,15,16,32}$ In contrast, isolates with mutations S450G, H445C, H445L and D435Y, belong to low-level or susceptible categories. In addition, several mutations, such as Q432K, Q432L, Q432P and S450F occurred exclusively in high-level RIF resistant isolates. For some mutations, however, the quantities of the isolates in this study are very small, which could affect the statistical power of our model. It is possible that the mutations are actually causing resistance, without being recognized by the statistical model.

RIF resistance is usually caused by mutations within RIF resistance-determining regions (RRDRs) of RpoB that are involved in the formation of the RIF-binding pocket. $^{35,36}$ Accordingly, all 11 mutated sites contributing to RIF-resistance in our study, including V170, Q429, L430, S431, Q432, D435, S441, H445, S450, L452 and I491, are located in the RRDRs of RpoB, ${ }^{35}$ while four residues outside RRDRs, namely P45, T400, G759 and Q1056, are not involved in RIF binding. Though in this study, mutations P45S, T400I, G759S and Q1056K occurred in isolates belonging to high-level RIF resistant group, these mutations were accompanied by additional mutations associated with high-level RIF resistance, S450L or H445Y. In addition, mutations on codons V170 and I491 were considered to lead to RIF resistance, which necessitated investigation beyond the RRDR, especially when patient treatment effects are not as expected.

Our analysis also showed that in the RRDRs, K446 and I488, do not directly interact with RIF and then mutations of these residues do not have the interaction with RIF. In addition, mutations Q429H, S431G and D435G do not have effect on interactions between wild-type RpoB and RIF. These contradictory results probably owe to using the different crystal structure models and different algorithms. ${ }^{35,36}$ Only by combining the results of structural prediction with phenotypic data could we determine whether a mutation contributes to drug resistance. However, in this study, these five mutations occurred in RIF-resistant isolates, and these mutations were accompanied by an additional RIF resistance-associated mutation. Interestingly, single mutation, for example, Q429H, was observed in both RIF-susceptible isolates and RIF-resistant isolates. ${ }^{6}$ Thus, several intra-RRDR mutations are considered not to involve in the development of RIF resistance. Instead, they may perform as compensatory mutations to alleviate fitness impairment incurred by other mutations directly associated with drug resistance. ${ }^{6}$ Further research might be required to confirm their functions. 
A

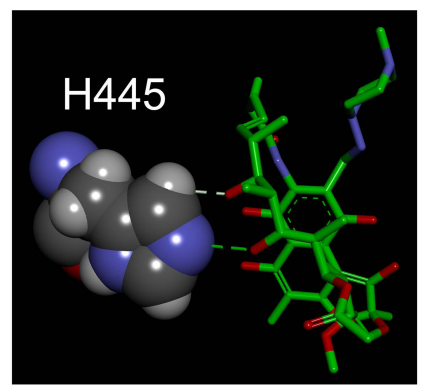

D

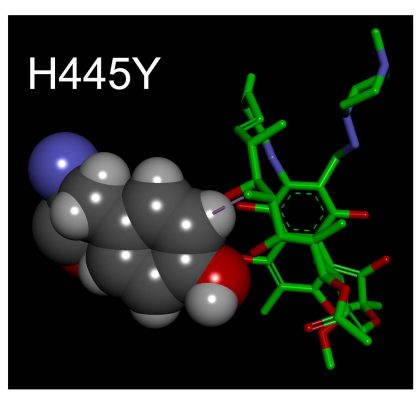

G

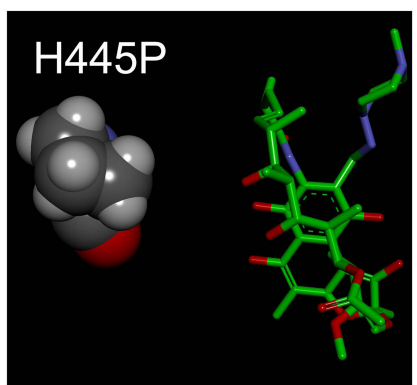

B

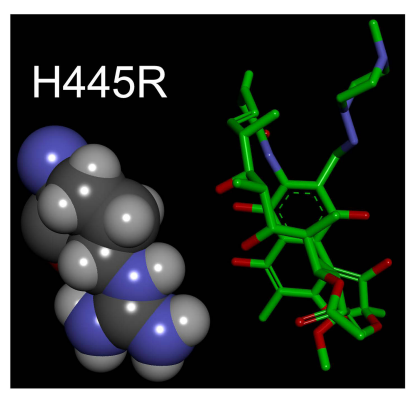

E

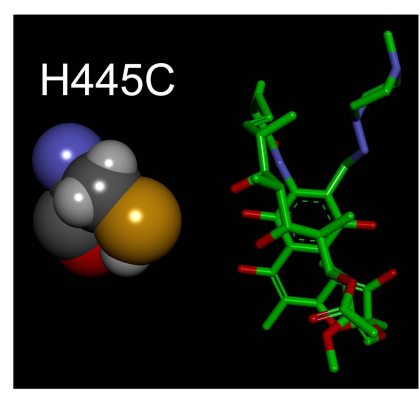

H

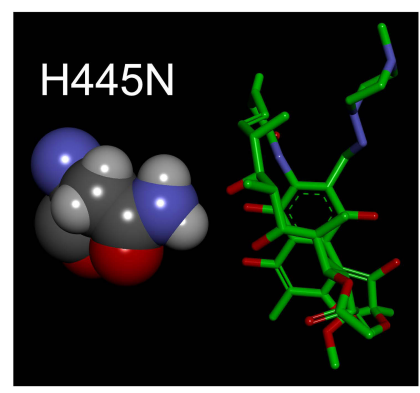

C

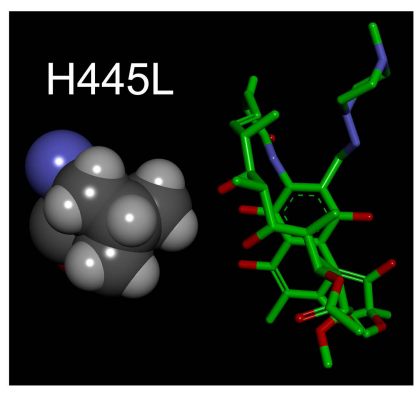

F

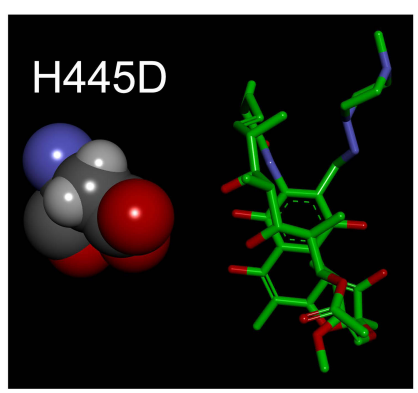

I

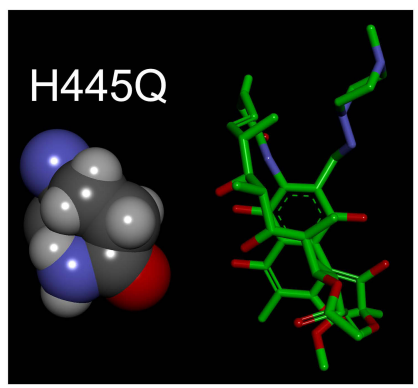

Figure 2 Interactions between rifampin and Residue 445 of wild-type/mutant RpoB. The detailed structure for rifampin (RIF) and Residue 445 of wild-type RpoB H445 (A), mutant H445R (B), mutant H445L (C), mutant H445Y (D), mutant H445C (E), mutant H445D (F), mutant H445P (G), mutant H445N (H) or mutant H445Q (I). Residue 445 of RpoB is shown in the left with a display style of Corey-Pauling-Koltun (CPK). The RIF molecule is shown in the right with a display style of stick. The intermolecular hydrogen bonds are indicated by green dashed lines.

We also tested some commonly “disputed" mutations, such as L430P, D435Y, D435N, D435L and L452P, which have been reported to confer highly discordant RIF results by culture-based drug susceptibility testing. ${ }^{31,34,37-39}$ Crystal structure analysis indicated that these mutations could disrupt the interaction between RpoB and RIF, and then lead to RIF resistance. MIC testing results also indicated that almost all these mutations occurred in RIF-resistant isolates. It is notable that single D435Y mutation was observed in only two isolates. One was a RIF-resistant isolate with a critical concentration MIC value $(1 \mu \mathrm{g} / \mathrm{mL})$. Another was RIF-susceptible isolate but with a MIC of $0.5 \mu \mathrm{g} / \mathrm{mL}$, which was suggested as the breakpoint for RIF in microplate-based assay to replace the existing one by a number of studies. ${ }^{38-41}$

There are a few RIF-resistant isolates without rрoB mutations reported in the prior studies. ${ }^{1,5,6,42}$ We also observed eight RIF-resistant isolates, including two highlevel RIF resistance isolates and six low-level RIF resistance isolates (three isolates had a MIC of $1 \mu \mathrm{g} / \mathrm{mL}$ and the other three isolates had MICs of 2, 4 and $8 \mu \mathrm{g} / \mathrm{mL}$, respectively), in the absence of mutations over the whole $r p o B$ gene. Phenotypic and genotype characteristics of these eight isolates were confirmed by retesting proportion method based on L-J medium and DNA sequencing 
A

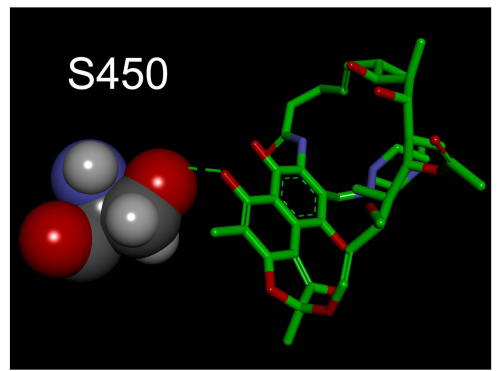

C

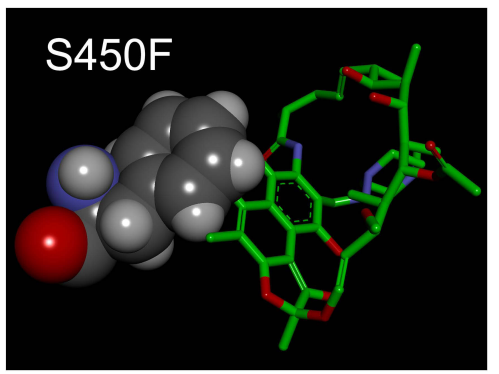

B

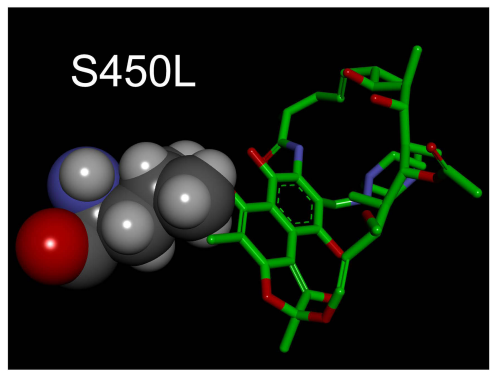

D

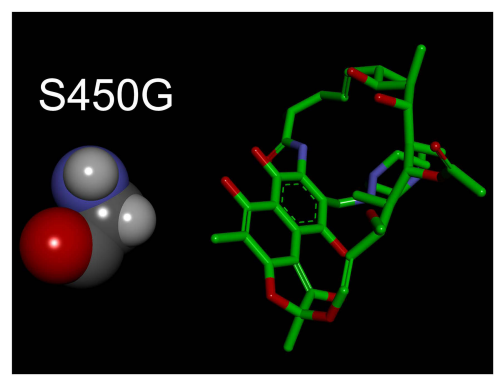

Figure 3 Interactions between rifampin and Residue 450 of wild-type/mutant RpoB. The detailed structure for rifampin (RIF) and Residue 450 of wild-type RpoB S450 (A), mutant S450L (B), mutant S450F (C) or mutant S450G (D). Residue 450 of RpoB is shown in the left with a display style of Corey-Pauling-Koltun (CPK). The RIF molecule is shown in the right with a display style of stick. The intermolecular hydrogen bonds are indicated by green dashed lines.

of $r p o B$. Whole-genome sequencing and genotype analysis of these eight isolates also showed that they derived from different origins (data not shown in this study). Resistance in these isolates might be attributed to other mechanisms, such as the upregulation of efflux pump, ${ }^{13,16}$ or the overexpression of transporter. ${ }^{43}$

However, there are limitations in this study. On the one hand, although 175 isolates represented a quite challenging panel of isolates in the studies on RIF-resistance mechanism so far, the number of isolates harboring certain types of $r p o B$ mutations was still limited. Also, some of the RIF resistant isolates could not be explained in the present study and clarifying them will further help us to reveal RIF resistance in M. tuberculosis.

In sum, we revealed a comprehensive profiles of mutations within the whole $r p o B$ gene and their associations with RIF resistance levels in a variety of M. tuberculosis clinical isolates. The effects of mutations on the interaction between RpoB and RIF were analyzed based on crystal structure. The information of these mutations and interactions will advance our mechanistic understanding of $M$. tuberculosis RIF- resistance and aid molecular testing and new drug design.

\section{Ethical Approval}

This study was approved by the Ethics Committee of National Institute for Communicable Disease Control and Prevention, Chinese Center for Disease Control and Prevention. All patients participating in the study had written consent from themselves.

\section{Funding}

This study was supported by the projects from National Key Program of Mega Infectious Diseases (Grant No. 2018ZX10302302-001) and National Basic Research Program of China (973 Program, Grant No. 2015CB554202). The funder had no role in the study design, data collection and analysis, decision to publish, or manuscript preparation.

\section{Disclosure}

All authors have no competing interests. 


\section{References}

1. World Health Organization. Global tuberculosis report 2020. Geneva, Switzerland: World Health Organization; 2020.

2. Campbell EA, Korzheva N, Mustaev A, et al. Structural mechanism for rifampicin inhibition of bacterial RNA polymerase. Cell. 2001;104:901-912.

3. Swain SS, Sharma D, Hussain T, Pati S. Molecular mechanisms of underlying genetic factors and associated mutations for drug resistance in Mycobacterium tuberculosis. Emerg Microbes Infect. 2020;9:1651-1663. doi:10.1080/22221751.2020.1785334

4. Campbell PJ, Morlock GP, Sikes RD, et al. Molecular detection of mutations associated with first- and second-line drug resistance compared with conventional drug susceptibility testing of Mycobacterium tuberculosis. Antimicrob Agents Chemother. 2011;55:2032-2041. doi:10.1128/AAC.01550-10

5. Jamieson FB, Guthrie JL, Neemuchwala A, Lastovetska O, Melano RG, Mehaffy C. Profiling of rpoB mutations and MICs for rifampin and rifabutin in Mycobacterium tuberculosis. $J$ Clin Microbiol. 2014;52:2157-2162. doi:10.1128/JCM.00691-14

6. Jagielski T, Bakula Z, Brzostek A, et al. Characterization of mutations conferring resistance to rifampin in Mycobacterium tuberculosis clinical strains. Antimicrob Agents Chemother. 2018;62:e1093-18. doi:10.1128/AAC.01093-18

7. Zaw MT, Emran NA, Lin Z. Mutations inside rifampicin-resistance determining region of rpoB gene associated with rifampicin-resistance in Mycobacterium tuberculosis. $J$ Infect Public Health. 2018;11:605-610. doi:10.1016/j.jiph.2018.04.005

8. Williams DL, Spring L, Collins L, et al. Contribution of rpoB mutations to development of rifamycin cross-resistance in Mycobacterium tuberculosis. Antimicrob Agents Chemother. 1998;42:1853-1857. doi:10.1128/AAC.42.7.1853

9. Ramaswamy S, Musser JM. Molecular genetic basis of antimicrobial agent resistance in Mycobacterium tuberculosis: 1998 update. Tuber Lung Dis. 1998;79:3-29. doi:10.1054/tuld.1998.0002

10. Rothman-Denes LB. Structure of Escherichia coli RNA polymerase holoenzyme at last. Proc Natl Acad Sci $U$ S A. 2013;110:19662-19663. doi:10.1073/pnas.1320604110

11. Andre E, Goeminne L, Cabibbe A, et al. Consensus numbering system for the rifampicin resistance-associated rpoB gene mutations in pathogenic mycobacteria. Clin Microbiol Infect. 2017;23:167-172. doi:10.1016/j.cmi.2016.09.006

12. Danilchanka O, Pires D, Anes E, Niederweis M. The Mycobacterium tuberculosis outer membrane channel protein CpnT confers susceptibility to toxic molecules. Antimicrob Agents Chemother. 2015;59:2328-2336. doi:10.1128/AAC.04222-14

13. Louw GE, Warren RM, Gey van Pittius NC, McEvoy CR, Van Helden PD, Victor TC. A balancing act: efflux/influx in mycobacterial drug resistance. Antimicrob Agents Chemother 2009;53:3181-3189. doi:10.1128/AAC.01577-08

14. Xu G, Liu H, Jia X, Wang X, Xu P. Mechanisms and detection methods of Mycobacterium tuberculosis rifampicin resistance: the phenomenon of drug resistance is complex. Tuberculosis. 2021;128:102083. doi:10.1016/j.tube.2021.102083

15. Ohno H, Koga H, Kohno S, Tashiro T, Hara K. Relationship between rifampin MICs for and rpoB mutations of Mycobacterium tuberculosis strains isolated in Japan. Antimicrob Agents Chemother. 1996;40:1053-1056. doi:10.1128/AAC.40.4.1053

16. Pang Y, Lu J, Wang Y, Song Y, Wang S, Zhao Y. Study of the rifampin monoresistance mechanism in Mycobacterium tuberculosis. Antimicrob Agents Chemother. 2013;57:893-900. doi:10.1128/AAC.01024-12

17. Berrada ZL, Lin SY, Rodwell TC, et al. Rifabutin and rifampin resistance levels and associated rpoB mutations in clinical isolates of Mycobacterium tuberculosis complex. Diagn Microbiol Infect Dis. 2016;85:177-181. doi:10.1016/j.diagmicrobio.2016.01.019
18. Wi YM, Greenwood-Quaintance KE, Brinkman CL, Lee JYH, Howden BP, Patel R. Rifampicin resistance in Staphylococcus epidermidis: molecular characterisation and fitness cost of rpoB mutations. Int J Antimicrob Agents. 2018;51:670-677. doi:10.1016/ j.ijantimicag.2017.12.019

19. World Health Organization. Guidelines for Surveillance of Drug Resistance in Tuberculosis. 4th ed. WHO/HTM/TB/2009.422. Geneva, Switzerland: World Health Organization; 2009.

20. Hall L, Jude KP, Clark SL, Wengenack NL. Antimicrobial susceptibility testing of Mycobacterium tuberculosis complex for first and second line drugs by broth dilution in a microtiter plate format. J Vis Exp. 2011. doi:10.3791/3094

21. Somerville W, Thibert L, Schwartzman K, Behr MA. Extraction of Mycobacterium tuberculosis DNA: a question of containment. $J$ Clin Microbiol. 2005;43:2996-2997. doi:10.1128/JCM.43.6.29962997.2005

22. Lin W, Mandal S, Degen D, et al. Structural basis of Mycobacterium tuberculosis transcription and transcription inhibition. Mol Cell. 2017;66:169-179 e8. doi:10.1016/j.molcel.2017.03.001

23. Farhat MR, Jacobson KR, Franke MF, et al. Gyrase mutations are associated with variable levels of fluoroquinolone resistance in Mycobacterium tuberculosis. J Clin Microbiol. 2016;54:727-733. doi:10.1128/JCM.02775-15

24. Sun Q, Xiao TY, Liu HC, et al. Mutations within embCAB are associated with variable level of ethambutol resistance in Mycobacterium tuberculosis isolates from China. Antimicrob Agents Chemother. 2018;62. doi:10.1128/AAC.01279-17

25. Artsimovitch I, Vassylyeva MN, Svetlov D, et al. Allosteric modulation of the RNA polymerase catalytic reaction is an essential component of transcription control by rifamycins. Cell. 2005;122:351-363. doi:10.1016/j.cell.2005.07.014

26. Friedrich SO, Venter A, Kayigire XA, Dawson R, Donald PR, Diacon AH. Suitability of Xpert MTB/RIF and genotype MTBDRplus for patient selection for a tuberculosis clinical trial. J Clin Microbiol. 2011;49:2827-2831. doi:10.1128/JCM.00138-11

27. Di Tanna GL, Khaki AR, Theron G, et al. Effect of Xpert MTB/RIF on clinical outcomes in routine care settings: individual patient data meta-analysis. Lancet Glob Health. 2019;7:e191-e199. doi:10.1016/ S2214-109X(18)30458-3

28. Xie YL, Chakravorty S, Armstrong DT, et al. Evaluation of a rapid molecular drug-susceptibility test for tuberculosis. $N$ Engl J Med. 2017;377:1043-1054. doi:10.1056/NEJMoa1614915

29. Atashi S, Izadi B, Jalilian S, Madani SH, Farahani A, Mohajeri P. Evaluation of GeneXpert MTB/RIF for determination of rifampicin resistance among new tuberculosis cases in west and northwest Iran. New Microbes New Infect. 2017;19:117-120. doi:10.1016/j. nmni.2017.07.002

30. Goldstein BP. Resistance to rifampicin: a review. J Antibiot. 2014;67 (9):625-630. doi:10.1038/ja.2014.107

31. Farhat MR, Sixsmith J, Calderon R, Hicks ND, Fortune SM, Murray M. Rifampicin and rifabutin resistance in 1003 Mycobacterium tuberculosis clinical isolates. $J$ Antimicrob Chemother. 2019;74:1477-1483. doi:10.1093/jac/dkz048

32. Shea J, Halse TA, Kohlerschmidt D, et al. Low-level rifampin resistance and rpoB mutations in Mycobacterium tuberculosis: an analysis of whole-genome sequencing and drug susceptibility test data in New York. J Clin Microbiol. 2020;59:e01885-20.

33. Mohajeri P, Sadri H, Farahani A, Norozi B, Atashi S. Frequency of mutations associated with rifampicin resistance in Mycobacterium tuberculosis strains isolated from patients in West of Iran. Microb Drug Resist. 2015;21:315-319. doi:10.1089/mdr.2014.0075

34. Nebenzahl-Guimaraes H, Jacobson KR, Farhat MR, Murray MB. Systematic review of allelic exchange experiments aimed at identifying mutations that confer drug resistance in Mycobacterium tuberculosis. J Antimicrob Chemother. 2014;69:331-342. doi:10.1093/jac/dkt358 
35. Ning Q, Wang D, Cheng F, Zhong Y, Ding Q, You J. Predicting rifampicin resistance mutations in bacterial RNA polymerase subunit beta based on majority consensus. BMC Bioinform. 2021;22:210. doi:10.1186/s12859-021-04137-0

36. Molodtsov V, Scharf NT, Stefan MA, Garcia GA, Murakami KS. Structural basis for rifamycin resistance of bacterial RNA polymerase by the three most clinically important RpoB mutations found in Mycobacterium tuberculosis. Mol Microbiol. 2017;103:1034-1045. doi: $10.1111 / \mathrm{mmi} .13606$

37. Van Deun A, Barrera L, Bastian I, et al. Mycobacterium tuberculosis strains with highly discordant rifampin susceptibility test results. J Clin Microbiol. 2009;47:3501-3506. doi:10.1128/JCM.01209-09

38. Rigouts L, Gumusboga M, de Rijk WB, et al. Rifampin resistance missed in automated liquid culture system for Mycobacterium tuberculosis isolates with specific rpoB mutations. J Clin Microbiol. 2013;51:2641-2645. doi:10.1128/JCM.02741-12

39. Miotto P, Cabibbe AM, Borroni E, Degano M, Cirillo DM. Role of disputed mutations in the rpoB gene in interpretation of automated liquid MGIT culture results for rifampin susceptibility testing of Mycobacterium tuberculosis. J Clin Microbiol. 2018;56. doi:10.1128/JCM.01599-17
40. Fowler PW. Epidemiological cutoff values for a 96-well broth microdilution plate for high throughput research antibiotic susceptibility testing of M. tuberculosis. medRxiv. 2021. doi:10.1101/ 2021.02.24.21252386

41. World Health Organization. Technical report on critical concentrations for drug susceptibility testing of isoniazid and the rifamycins (rifampicin, rifabutin and rifapentine). World Health Organization; 2021. Available from: https://apps.who.int/iris/handle/10665/339275. Accessed September 22, 2021.

42. Comas I, Borrell S, Roetzer A, et al. Whole-genome sequencing of rifampicin-resistant Mycobacterium tuberculosis strains identifies compensatory mutations in RNA polymerase genes. Nat Genet. 2011;44:106-110. doi:10.1038/ng.1038

43. Sharma D, Bisht D, Khan AU. Potential alternative strategy against drug resistant tuberculosis: a proteomics prospect. Proteomes. 2018;6 (2):26. doi:10.3390/proteomes6020026

\section{Publish your work in this journal}

Infection and Drug Resistance is an international, peer-reviewed openaccess journal that focuses on the optimal treatment of infection (bacterial, fungal and viral) and the development and institution of preventive strategies to minimize the development and spread of resistance. The journal is specifically concerned with the epidemiology of antibiotic resistance and the mechanisms of resistance development and diffusion in both hospitals and the community. The manuscript management system is completely online and includes a very quick and fair peerreview system, which is all easy to use. Visit http://www.dovepress.com/ testimonials.php to read real quotes from published authors. 